\title{
Recurrent Urinary Tract Infections in Children With Bladder and Bowel Dysfunction
}

Nader Shaikh, MD, MPH, ${ }^{\mathrm{a}}$ Alejandro Hoberman, MD, ${ }^{\text {a }}$ Ron Keren, MD, ${ }^{\mathrm{b}}$ Nathan Gotman, MS, ${ }^{\mathrm{c}}$ Steven G. Docimo, MD, ${ }^{d}$ Ranjiv Mathews, MD, ${ }^{e}$ Sonika Bhatnagar, MD, MPH, ${ }^{a}$ Anastasia Ivanova, $\mathrm{PhD},{ }^{\mathrm{c}}$ Tej K. Mattoo, MD, FRCP, ${ }^{f}$ Marva Moxey-Mims, MD, ${ }^{8}$ Myra A. Carpenter, PhD, ${ }^{\mathrm{c}}$ Hans G. Pohl, MD, ${ }^{\text {h }}$ Saul Greenfield, MDi

BACKGROUND: Little generalizable information is available on the outcomes of children diagnosed with bladder and bowel dysfunction (BBD) after a urinary tract infection (UTI). Our objectives were to describe the clinical characteristics of children with BBD and to examine the effects of BBD on patient outcomes in children with and without vesicoureteral reflux (VUR).

METHODS: We combined data from 2 longitudinal studies (Randomized Intervention for Children With Vesicoureteral Reflux and Careful Urinary Tract Infection Evaluation) in which children $<6$ years of age with a first or second UTI were followed for 2 years. We compared outcomes for children with and without BBD, children with and without VUR, and children with VUR randomly assigned to prophylaxis or placebo. The outcomes examined were incidence of recurrent UTIs, renal scarring, surgical intervention, resolution of VUR, and treatment failure.

RESULTS: BBD was present at baseline in 54\% of the 181 toilet-trained children included; $94 \%$ of children with BBD reported daytime wetting, withholding maneuvers, or constipation. In children not on antimicrobial prophylaxis, 51\% of those with both BBD and VUR experienced recurrent UTIs, compared with $20 \%$ of those with VUR alone, $35 \%$ with BBD alone, and 32\% with neither BBD nor VUR. BBD was not associated with any of the other outcomes investigated.

concLusions: Among toilet-trained children, those with both BBD and VUR are at higher risk of developing recurrent UTIs than children with isolated VUR or children with isolated BBD and, accordingly, exhibit the greatest benefit from antimicrobial prophylaxis.

Divisions of ${ }^{a}$ General Academic Pediatrics, and dUrology, University of Pittsburgh School of Medicine, Children's Hospital of Pittsburgh of UPMC, Pittsburgh, Pennsylvania; ${ }^{b}$ Division of General Pediatrics, Center for Pediatric Clinical Effectiveness, Children's Hospital of Philadelphia, Philadelphia, Pennsylvania; ${ }^{C}$ Collaborative Studies Coordinating Center, Department of Biostatistics, University of North Carolina at Chapel Hill, Chapel Hill, North Carolina; ${ }^{e}$ Division of Urology, Southern Illinois University School of Medicine, Springfield, Illinois; ${ }^{f}$ Children's Hospital of Michigan, Wayne State University, Detroit, Michigan; ${ }^{g}$ National Institute of Diabetes, and Digestive and Kidney Diseases, Bethesda, Maryland; ' $D$ Division of Urology, Children's National Medical Center, George Washington University School of Medicine, Washington, District of Columbia; and 'Division of Pediatric Urology, State University of New York at Buffalo School of Medicine and Biomedical Sciences, Buffalo, New York

Dr Shaikh conceptualized the study concept and design and participated in data acquisition and drafting of the initial manuscript; Drs Hoberman, Keren, Docimo, Mathews, Bhatnagar, Mattoo, Moxey-Mims, Pohl, and Greenfield participated in data acquisition and drafting of the initial manuscript; Mr Gotman, Ms Ivanova, and Ms Carpenter participated in data analysis and drafting of the initial manuscript; and all authors approved the final manuscript as submitted.

DOI: $10.1542 /$ peds.2015-2982
WHAT'S KNOWN ON THIS SUBJECT: Few studies have examined the long-term outcomes of children with bladder and bowel dysfunction (BBD). Furthermore, little is known about the interaction between BBD and vesicoureteral reflux (VUR). We examined the sequelae of BBD in children with and without VUR.

WHAT THIS STUDY ADDS: We found that children with both BBD and VUR are at higher risk of developing recurrent urinary tract infections than children with isolated VUR or children with isolated BBD. These findings have important implications for the screening and treatment of VUR.

To cite: Shaikh N, Hoberman A, Keren R, et al. Recurrent Urinary Tract Infections in Children With Bladder and Bowel Dysfunction. Pediatrics. 2016;137(1):e20152982 
TABLE 1 Characteristics and Results of Previous Studies Reporting Outcomes of Children With BBD Presented in Reverse Chronologic Order

\begin{tabular}{|c|c|c|c|c|c|c|c|c|c|}
\hline Author, y & $N$ & Age (y) & $\begin{array}{l}\text { Inclusion } \\
\text { Criteria }\end{array}$ & $\begin{array}{c}\text { BBD } \\
\text { Prevalence }\end{array}$ & BBD Definition & Study Design & $\begin{array}{c}\text { BBD } \\
\text { Associated } \\
\text { With UTI } \\
\text { Recurrence }\end{array}$ & $\begin{array}{l}\text { BBD } \\
\text { Delayed VUR } \\
\text { Resolution }\end{array}$ & $\begin{array}{l}\text { BBD } \\
\text { Associated } \\
\text { With Renal } \\
\text { Scarring }\end{array}$ \\
\hline $\begin{array}{c}\text { Koff et al, }{ }^{1} \\
1998\end{array}$ & 143 & - & VUR & $43 \%$ & Clinical & $\begin{array}{l}\text { Convenience sample of } \\
\text { children whose VUR resolved } \\
\text { or was surgically corrected }\end{array}$ & Yes & Yes & - \\
\hline $\begin{array}{l}\text { Naseer and } \\
\text { Steinhardt, } 2 \\
1997\end{array}$ & 2100 & - & UTI & $>38 \%^{\mathrm{a}}$ & $\begin{array}{c}\text { Daytime } \\
\text { incontinence }\end{array}$ & $\begin{array}{l}\text { Prospective database of } \\
\text { children with UTI in clinical } \\
\text { practice }\end{array}$ & - & - & Yes \\
\hline $\begin{array}{l}\text { van Gool } \\
\quad \text { et al, }{ }^{3} 1992\end{array}$ & 386 & - & VUR & $18 \%$ & Questionnaire & $\begin{array}{l}\text { Prospective randomized trial } \\
\text { of prophylaxis vs surgery; } \\
\text { children with overt } \\
\text { symptoms of BBD excluded }\end{array}$ & Yes & Yes & - \\
\hline $\begin{array}{c}\text { Snodgrass, } \\
1991\end{array}$ & 109 & $0.1-18$ & UTI or BBD & $41 \%$ & Clinical & Cross-sectional & $\begin{array}{c}\text { BBD } \\
\text { associated } \\
\text { with } \\
\text { previous } \\
\text { UTI }\end{array}$ & - & - \\
\hline Seruca, ${ }^{5} 1989$ & 101 & $0.5-8$ & $\begin{array}{l}\text { VUR and } \\
\text { BBD }\end{array}$ & - & Unclear & $\begin{array}{l}\text { Prospective cohort ( } n=53 \text { ) } \\
\text { with treated BBD compared } \\
\text { with historical cohort ( } n= \\
\text { 48) with untreated BBD }\end{array}$ & - & $\begin{array}{l}\text { Yes, but no } \\
\text { correction } \\
\text { for baseline } \\
\text { differences in } \\
\text { the } 2 \text { cohorts }\end{array}$ & - \\
\hline
\end{tabular}

Dash indicates that data were not reported in the article.

${ }^{a}$ Calculated with total number of children in the study as the denominator; however, some children in the study were not toilet trained.

Few studies have prospectively characterized the outcomes of children with urinary tract infection (UTI) according to the presence or absence of bladder and bowel dysfunction (BBD). In many of the existing studies (Table 1), enrollment was restricted to certain highrisk subgroups. Accordingly, little generalizable information is available about the outcomes of children with BBD.

Our objectives were to examine the clinical characteristics and prevalence of BBD in a wellcharacterized population of toilet-trained children with UTI, to determine how BBD affects patient outcomes, to examine interactions between BBD and vesicoureteral reflux (VUR) on patient outcomes, and to determine whether the efficacy of antimicrobial prophylaxis varies between children with and without BBD.

\section{METHODS}

For this report, we combined data from 2 longitudinal studies (the Randomized Intervention for Children With Vesicoureteral Reflux [RIVUR] trial and the Careful Urinary Tract Infection Evaluation [CUTIE] study). Of the 802 total children enrolled in these studies, we excluded children who were not toilet trained at baseline $(N=606)$ and children with missing data for baseline $\operatorname{BBD}(n=13)$ or race $(n=2)$. The final analytical sample included 181 children. The methods of the RIVUR trial have been described previously. ${ }^{6}$ Briefly, we enrolled children aged 2 to 71 months of age presenting with grades I to IV VUR diagnosed after a first or second UTI at 19 clinical centers throughout North America. Children in the RIVUR trial were randomly assigned to antimicrobial prophylaxis or placebo. Children without VUR from 3 of the 19 RIVUR sites (Pittsburgh, Washington, DC, and Philadelphia) were enrolled in the parallel CUTIE study. ${ }^{7}$ The follow-up of children in both studies was identical, with the exception that children in the RIVUR trial had a dimercaptosuccinic acid (DMSA) renal scan at the 12-month follow-up visit and had a voiding cystourethrogram (VCUG) at the 24-month follow-up visit. Children in the CUTIE study received no antimicrobial prophylaxis. The 3 groups compared in this article were the RIVUR placebo, RIVUR prophylaxis, and CUTIE cohorts.

To assess BBD, we administered the Dysfunctional Voiding Scoring System (DVSS) ${ }^{8}$ questionnaire to parents of children who were reportedly fully toilet trained (for both bladder and bowel). The DVSS was administered at enrollment and at the 1- and 2-year follow-up visits. The DVSS is a previously validated 10-item scale (total score range $0-30$ ) that asks about the presence of bladder and bowel symptoms over the previous month. The majority of items are rated on a 0 to 3 scale with the following response categories: almost never (0), less than half the time (1), about half the time (2), and almost every time (3). In this study, we used the cutoffs recommended by the developers: Girls with a score of $\geq 6$ and boys with a score of $\geq 9$ were considered to have BBD. A 
symptom was considered absent when the score for that item was 0 . We modified the wording of a few questions to make them more easily understandable for parents with lower literacy levels. This modified version was successfully piloted in an earlier study. ${ }^{9}$ Participants with $\geq 3$ missing items on the DVSS were excluded from all analyses. The age of bladder and bowel training was the age the parent reported that the child began urinating and defecating in the toilet or potty. We asked parents about toilet training every 2 months.

The Paris Consensus on Childhood Constipation Terminology (PACCT) 6-item questionnaire asked about the occurrence of 6 key symptoms and signs of constipation $(<3$ bowel movements per week, $\geq 1$ episode of fecal incontinence per week, large stools obstructing the toilet, stoolwithholding behaviors, pain with bowel movements, and palpable stool on abdominal examination) over the previous 8 weeks. ${ }^{10} \mathrm{We}$ used a modified 5 -item version of this scale (omitting the item regarding the presence of palpable stool on abdominal examination). The presence of $\geq 2$ symptoms on this modified scale was considered evidence of constipation. The PACCT questionnaire was administered at baseline, 12 months, and 2 years.

The following outcomes were considered: recurrent UTIs, renal scarring, treatment failure, resolution of VUR, and surgery for VUR. Any child with $\geq 1$ UTI during the 2-year follow-up period was considered to have recurrent UTIs. Renal scarring was defined as the presence of photopenia plus contour change on an outcome DMSA scan performed after 2 years of follow-up or 3 to 4 months after treatment failure. Treatment failure was defined as the occurrence of 2 febrile UTIs, 1 febrile UTI and 3 symptomatic UTIs, 4 symptomatic UTIs, or new or worsening renal scarring. Resolution of VUR was defined as the absence of
VUR on the VCUG performed after 2 years of follow-up. Treatment failure, resolution of VUR, and surgery for VUR were assessed only for children in the RIVUR trial.

We defined 2 versions of BBD: BBD at baseline and BBD over time. For the latter, we included children not toilet trained at baseline, for a total of $n=785$ children. To calculate BBD over time, we divided each child's follow-up period into 3 intervals. During each interval, 4 states were possible: BBD present, BBD absent, not toilet trained, and toilet trained but BBD unknown. The BBD status during the first 6-month follow-up period was determined based on the BBD status at enrollment. The BBD status during months 6 to 18 and 18 to 24 was determined based on the BBD status at 1 year and 2 years, respectively. Time periods where BBD status was unknown were excluded from the analyses.

For time-to-event outcomes (UTI, treatment failure), we computed unadjusted and adjusted hazard ratios (HRs) and $P$ values by using Cox proportional hazards regression. Adjusted models were stratified by administrative site (6 categories). For binary outcomes (scarring, resolution of VUR), we used logistic regression to calculate unadjusted odds ratios (ORs) and $P$ values. For surgery, because of the low number of events we used Fisher's exact test to calculate $P$ values. Children who did not have an outcome VCUG or DMSA scan were excluded from the aforementioned analysis.

To assess differential effects of treatment and the differential effects of VUR in the BBD subgroups, we used a Cox regression model that included the interaction between study group (RIVUR placebo, RIVUR prophylaxis, or CUTIE) and BBD.

\section{RESULTS}

Table 2 describes clinical and demographic characteristics of the
181 children included. BBD was present at baseline in 97 (54\%) of the children. The CUTIE study group had a higher proportion of Hispanic children $(21 \%$ vs $6 \%$ vs $8 \%$ in CUTIE, RIVUR prophylaxis, and RIVUR placebo, respectively) and a lower proportion of children with a febrile index UTI ( $40 \%$ vs $58 \%$ vs $69 \%$, respectively).

At baseline, the most frequent urinary symptoms reported in children with BBD were urinary urgency in 82 (85\%), withholding maneuvers (ie, pee dance, squatting, or crossing legs) in 77 (80\%), and daytime wetting in 60 (63\%); 88 (92\%) reported either daytime wetting or withholding maneuvers. Among children with BBD at baseline, 38 (39\%) reported frequent painful defecation and $8(8 \%)$ reported $<3$ bowel movements per week over the last 8 weeks; 21 (22\%) met criteria for constipation according to the modified PACCT criteria. Ninety children (94\%) with BBD at baseline reported daytime wetting, withholding maneuvers, or constipation (PACCT criteria). Of the 47 children with none of these symptoms, only 6 (13\%) had BBD.

No significant differences were noted in median age, race, ethnicity, primary caregiver education level, UTI type, and history of UTI in children with and without BBD. The minimum age of children with no BBD was 24 months; $75 \%$ were $>40$ months. The minimum age of children with BBD was 26 months; $75 \%$ were $>41$ months. No boys (0/4) met criteria for BBD, compared with 97 out of 177 girls (55\%, Fisher's exact test $P=.04$ ). Seventyone of 124 toilet-trained children with VUR (57\%) had BBD, compared with 26 out of 57 (46\%) of children without VUR $(P=.15)$. Among toilet-trained children with VUR, the proportions with BBD were similar across VUR grades $(44 \%-64 \%, P=$ $.64)$. None of the specific symptoms 


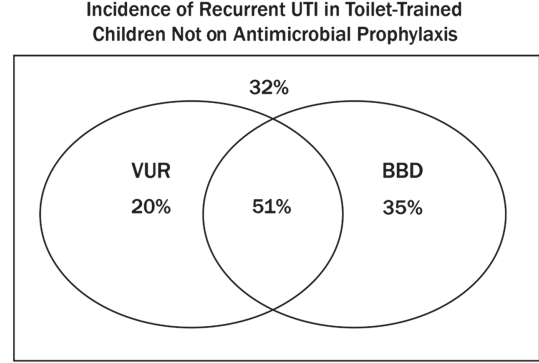

FIGURE 1

Incidence of recurrent UTIs according to the presence of BBD and VUR in toilet-trained children with UTI who are not receiving antimicrobial prophylaxis

of BBD or constipation were clearly associated with VUR.

Outcomes of children with and without BBD at baseline are described in Table 3. In the RIVUR placebo cohort, BBD was associated with a higher risk of recurrent UTIs; $51 \%$ of children with BBD had a recurrent UTI, compared with $20 \%$ of children without BBD (HR = 3.49; 95\% confidence interval [CI], 1.30-9.38) (Fig 1). This association was even larger $(\mathrm{HR}=5.71 ; 95 \% \mathrm{CI}$, 1.94-16.78) after we adjusted for age, gender, and race and stratified by site. In contrast, in the RIVUR prophylaxis and CUTIE cohorts, the rates of recurrent UTIs were similar among children with and without BBD $(18 \%$ vs $25 \%$ in the RIVUR prophylaxis group and $35 \%$ vs $32 \%$ in the CUTIE group, for the BBD and no BBD subgroups, respectively). For children without BBD, the UTI rate was slightly higher in children without VUR (32\%) than in children with VUR (20\%). In all 3 cohorts, BBD was not associated with renal scarring. In both RIVUR cohorts, BBD was not associated with VUR resolution or surgery for VUR. However, because of the rarity of these events in our cohort, we had limited power to detect differences for these outcome measures.

Results were similar when we used the BBD over time variable (Supplemental Table 4); BBD was associated with recurrent UTIs in the

TABLE 2 Demographic and Clinical Characteristics of Study Population at the Time of Enrollment

\begin{tabular}{|c|c|c|c|c|}
\hline Characteristic & $\begin{array}{c}\text { RIVUR } \\
\text { Prophylaxis } \\
(N=62)\end{array}$ & $\begin{array}{l}\text { RIVUR } \\
\text { Placebo ( } N \\
=62)\end{array}$ & $\begin{array}{c}\text { CUTIE }(N= \\
57)\end{array}$ & $p^{a}$ \\
\hline Median age (IQR), mo & $45(36-56)$ & $47(41-58)$ & $47(42-57)$ & .43 \\
\hline Female, \% & 100 & 97 & 96 & .47 \\
\hline Hispanic, \% & 6 & 8 & 21 & .04 \\
\hline Nonwhite race, \% & 18 & 21 & 25 & .63 \\
\hline Primary caregiver education & & & & .23 \\
\hline High school graduate or less, $\%$ & 24 & 16 & 33 & \\
\hline Some college, \% & 21 & 29 & 18 & \\
\hline College or higher, \% & 55 & 55 & 49 & \\
\hline Median age (IQR) of toilet training, mo & $28(24-33)$ & $30(24-36)$ & $30(24-36)$ & .11 \\
\hline Index UTI febrile, \% & 58 & 69 & 40 & .007 \\
\hline$>1$ UTI in the past, $\%$ & 29 & 18 & 26 & .30 \\
\hline VUR at baseline & & & & $<.001$ \\
\hline None, \% & 0 & 0 & 100 & \\
\hline Grade $1, \%$ & 16 & 16 & 0 & \\
\hline Grade 2, \% & 45 & 50 & 0 & \\
\hline Grade $3, \%$ & 31 & 27 & 0 & \\
\hline Grade 4, \% & 8 & 6 & 0 & \\
\hline Constipation per PACCT at baseline, $\%$ & 13 & 13 & 14 & $>.99$ \\
\hline BBD at baseline, \% & 55 & 60 & 46 & .31 \\
\hline
\end{tabular}

IQR, interquartile range.

a $P$ values were computed with the $\chi^{2}$ test for VUR, Fisher's exact test for other categorical variables, and the KruskalWallis test for continuous variables.

TABLE 3 Clinical Outcomes of Children With UTI According to the Presence or Absence of BBD at Baseline

\begin{tabular}{|c|c|c|c|c|c|c|c|}
\hline \multirow[t]{2}{*}{ Group and Outcome } & \multicolumn{2}{|c|}{ BBD } & \multicolumn{2}{|c|}{ No BBD } & \multicolumn{3}{|c|}{ BBD vs No BBD } \\
\hline & $\%$ & $n / N$ & $\%$ & $n / N$ & $\begin{array}{l}\text { OR or } \\
\text { HR }\end{array}$ & $95 \% \mathrm{Cl}$ & $P$ \\
\hline \multicolumn{8}{|l|}{$\begin{array}{l}\text { VUR, on prophylaxis (RIVUR } \\
\text { prophylaxis) }\end{array}$} \\
\hline Recurrent UTI ${ }^{\mathrm{a}}$ & 18 & $6 / 34$ & 25 & $7 / 28$ & 0.69 & $0.23-2.07$ & .51 \\
\hline Recurrent febrile UTIa & 9 & $3 / 34$ & 14 & $4 / 28$ & 0.63 & $0.14-2.80$ & .54 \\
\hline Treatment failure ${ }^{a}$ & 3 & $1 / 34$ & 11 & $3 / 28$ & 0.28 & $0.03-2.68$ & .27 \\
\hline Renal scarring ${ }^{b}$ & 25 & $6 / 24$ & 15 & $4 / 27$ & 1.92 & $0.47-7.83$ & .36 \\
\hline VUR resolved by year $2^{b}$ & 46 & $12 / 26$ & 41 & $9 / 22$ & 1.24 & $0.39-3.90$ & .72 \\
\hline Surgery for VURC & 0 & $0 / 34$ & 4 & $1 / 28$ & - & - & .45 \\
\hline \multicolumn{8}{|l|}{$\begin{array}{l}\text { VUR, no prophylaxis (RIVUR } \\
\text { placebo) }\end{array}$} \\
\hline Recurrent UTIa & 51 & $19 / 37$ & 20 & $5 / 25$ & 3.49 & $1.30-9.38$ & .01 \\
\hline Recurrent febrile UTI & 27 & $10 / 37$ & 16 & $4 / 25$ & 2.08 & $0.65-6.65$ & .22 \\
\hline Treatment failure ${ }^{a}$ & 19 & $7 / 37$ & 8 & $2 / 25$ & 2.89 & $0.60-13.92$ & .19 \\
\hline Renal scarring ${ }^{b}$ & 21 & $6 / 29$ & 22 & $5 / 23$ & 0.94 & $0.25-3.58$ & .93 \\
\hline VUR resolved by year $2^{\mathrm{b}}$ & 46 & $12 / 26$ & 53 & $9 / 17$ & 0.76 & $0.22-2.59$ & .66 \\
\hline Surgery for VURC & 8 & $3 / 37$ & 0 & $0 / 25$ & - & - & .27 \\
\hline \multicolumn{8}{|l|}{$\begin{array}{l}\text { No VUR, no prophylaxis } \\
\text { (CUTIE) }\end{array}$} \\
\hline Recurrent UTIa & 35 & $9 / 26$ & 32 & $10 / 31$ & 1.10 & $0.45-2.70$ & .84 \\
\hline Recurrent febrile UTI & 19 & $5 / 26$ & 13 & $4 / 31$ & 1.49 & $0.40-5.57$ & .55 \\
\hline Renal scarringb & 10 & $2 / 20$ & 14 & $3 / 22$ & 0.70 & $0.11-4.71$ & .72 \\
\hline
\end{tabular}

Dash indicates not calculable; $n / N=$ number of events/number of evaluable patients.

${ }^{\text {a }}$ HR for time to event is reported.

${ }^{b} 0$ Rs are reported.

c Fisher's exact test was used to compute $P$ values for VUR surgery.

RIVUR placebo cohort but not in the RIVUR prophylaxis or CUTIE cohorts. In the RIVUR placebo cohort, risk of recurrent UTI during times with BBD was 3 times the risk during times without BBD. 
Among children with BBD, the proportion of children with recurrent UTIs in the RIVUR prophylaxis group was lower (18\%) than in the RIVUR placebo group (51\%). In contrast, among children without BBD, the proportion of children with recurrent UTIs in the RIVUR prophylaxis group (25\%) and the RIVUR placebo group (20\%) were similar. We used a Cox regression model with a $\mathrm{BBD}-$ study group interaction and found that this association between use of antimicrobial prophylaxis and recurrent UTIs was significantly stronger $(P$ value for interaction $=$ .04 ) in children with BBD (HR = 0.22; 95\% CI, 0.08-0.61) than in children without BBD (HR = 1.46; 95\% CI, 0.45-4.79).

With regard to the impact of VUR on BBD subgroups, compared with the RIVUR placebo group, the CUTIE group had a slightly lower proportion of children with recurrent UTIs among those with BBD (35\%) but a slightly higher proportion of children with recurrent UTIs among those with no BBD (32\%). In the time-toevent model, corresponding HRs for VUR were 2.08 (95\% CI, 0.85-5.09) for the BBD group and 0.60 for the no BBD group (95\% CI, 0.19-1.85).

Of note, grade of VUR appeared not to appreciably modify the association between $\mathrm{BBD}$ and recurrent UTIs. Among children with BBD in the RIVUR placebo group, $50 \%$ of children with VUR grades I or II and $54 \%$ of children with VUR grades III or IV had recurrent UTIs. The corresponding numbers for children with no BBD in the RIVUR placebo group were $24 \%$ and $13 \%$.

\section{DISCUSSION}

Data presented in this article provide a unique window into the poorly understood relationship between VUR, BBD, antimicrobial prophylaxis, and recurrent UTIs. Children with both VUR and BBD had the highest rate of recurrent UTIs, whereas children with only BBD or only VUR had no evidence of a higher risk compared with children with neither. Our results also underscore that, among toilet-trained children, prophylaxis was significantly more effective in children with both VUR and BBD than in any other subgroup of children. This pattern suggests that children with both BBD and VUR have a substantial risk of recurrent UTIs and could potentially benefit the most from antimicrobial prophylaxis.

\section{What Is the Prevalence of BBD in Children With UTI?}

The prevalence of BBD in our sample (54\%) is clearly higher than what has been reported in the general population (20\%), ${ }^{9}$ probably because of the association between BBD and UTI. Our results are consistent with those of previous studies of children with UTI. ${ }^{4}$

\section{Are BBD and VUR Causally Related?}

Several studies have reported that the prevalence of BBD in children referred to urology clinics for VUR is high (34\%-70\%). ${ }^{1,11-13}$ Perhaps because of these studies, the importance of BBD is well recognized among urologists. The high prevalence of BBD in children with VUR has led some to assume that VUR and BBD are causally related. We found that BBD often is present in children with UTI regardless of the presence of VUR, which suggests that BBD and VUR are not causally related.

\section{What Are the Practical Implications of These Findings?}

Because the risk of recurrent UTI is particularly high among children who have both BBD and VUR, it seems logical to screen for $\geq 1$ of these conditions in children diagnosed with UTI (provided that costeffective screening and treatment strategies are available). With proper training, screening for BBD could be performed by primary care physicians. In contrast, definitive screening for VUR requires invasive testing. Similarly, treatment of BBD is arguably less likely to cause harm than treatment of VUR; the former usually requires use of laxatives, timed voiding, or biofeedback ${ }^{14}$, whereas the latter requires using long-term antimicrobial prophylaxis or surgery. Thus, one approach would be to routinely screen all toilet-trained children with UTI for $\mathrm{BBD}$ and to treat BBD in those who screen positive. Determining whether such an approach is more effective than other approaches (eg, VCUG in children with BBD, VCUG in children with a second UTI or in children with an abnormal ultrasound, VCUG in children with a high biomarker level) requires additional study.

Until such studies are conducted, our results suggest that screening for BBD is an important part of caring for children with UTIs, especially given the high prevalence of BBD in this population. In a previous study, we showed that BBD is often overlooked and rarely treated by general practitioners. ${ }^{9}$ When faced with a child with symptoms of BBD (eg, with recurrent episodes of urgency and incontinence), many clinicians repeatedly test for UTI, failing to recognize BBD as the underlying cause of the child's symptoms. These episodes represent missed opportunities to diagnose and treat BBD. Neither the diagnosis nor treatment of BBD requires specialist input. General practitioners could easily ask about constipation and treat it when it is present. In addition to asking about constipation, physicians should ask about daytime wetting and withholding behaviors. If these symptoms are present, timed voiding and double voiding (having the child sit again on the toilet right after he or she has finished voiding) may help alleviate them. In children who do not have daytime wetting, withholding behaviors, 
or constipation, no additional assessment would be needed. Alternatively, the DVSS, which was used as the screening tool in this study and takes 2 minutes to complete, could be used in primary care offices as a screening tool.

Several limitations are noteworthy. First, we reached some of our conclusions by comparing 3 cohorts of children enrolled in 2 related studies. Of the 3 cohorts, 2 represent arms of a randomized controlled trial (RIVUR). The CUTIE study differed demographically from the other cohorts, in part because enrollment in the CUTIE study was limited to 3 of the 19 sites participating in RIVUR. However, the study procedures were almost identical in both studies. Second, the number of toilet-trained children was too few to evaluate some of the less frequently occurring secondary outcomes (eg, scarring, surgery). Third, because of the small number of boys included, our results apply primarily to girls. Fourth, although our data suggest that BBD is an important risk factor for recurrent UTIs, future studies must be conducted to determine whether screening and treatment of BBD actually improve patient outcomes. Finally, analyses conducted here were not prespecified in the study protocol. Nevertheless, our study is unique in that children with UTIs were systematically included from a wide network of practices, including many primary care practices.

All children had complete and consistent anatomic evaluation. BBD was assessed through a validated questionnaire, and outcomes associated with the presence of BBD were examined longitudinally.

In conclusion, BBD is not limited to children with VUR; it is present in $\sim 50 \%$ of children with UTIs. Children with both BBD and VUR are at highest risk of developing recurrent UTIs and receive the greatest benefit from antimicrobial prophylaxis. Screening and treatment of BBD may reduce recurrent UTIs. For many years, physicians caring for children with UTI have focused their efforts on identifying and managing VUR. This report highlights the importance of BBD in the pathogenesis of recurrent UTIs and clearly signals a need to broaden our attention beyond the identification of VUR.

\section{ACKNOWLEDGMENTS}

The authors thank Drs Gordon McLorie and Russell W. Chesney for their contributions to the RIVUR study and this manuscript.

\section{ABBREVIATIONS}

BBD: bladder and bowel dysfunction

CI: confidence interval

CUTIE: Careful Urinary Tract Infection Evaluation

DMSA: dimercaptosuccinic acid

DVSS: Dysfunctional Voiding Scoring System

HR: hazard ratio

OR: odds ratio

PACCT: Paris Consensus on Childhood Constipation Terminology

RIVUR: Randomized Intervention for Children With Vesicoureteral Reflux

UTI: urinary tract infection VCUG: voiding cystourethrogram VUR: vesicoureteral reflux

Accepted for publication 0ct 7, 2015

Address correspondence to Nader Shaikh, MD, MPH, Children's Hospital of Pittsburgh of UPMC, One Children's Hospital Drive, 4401 Penn Ave, Pittsburgh, PA 15224. E-mail: nader.shaikh@chp.edu

PEDIATRICS (ISSN Numbers: Print, 0031-4005; Online, 1098-4275).

Copyright (C) 2016 by the American Academy of Pediatrics

FINANCIAL DISCLOSURE: The authors have indicated they have no financial relationships relevant to this article to disclose.

FUNDING: The content is solely the responsibility of the authors and does not necessarily represent the official views of the National Institute of Diabetes and Digestive and Kidney Diseases or the National Institutes of Health. This research was supported by grants U01 DK074059, U01 DK074053, U01 DK074082, U01 DK074064, U01 DK074062, and U01 DK074063 from the National Institute of Diabetes and Digestive and Kidney Diseases, National Institutes of Health, Department of Health and Human Services. Funded by the National Institutes of Health (NIH).

POTENTIAL CONFLICT OF INTEREST: The authors have indicated they have no potential conflicts of interest to disclose.

\section{REFERENCES}

1. Koff SA, Wagner TT, Jayanthi VR. The relationship among dysfunctional elimination syndromes, primary vesicoureteral reflux and urinary tract infections in children. $J$ Urol. 1998;160 (3 pt 2):1019-1022

2. Naseer SR, Steinhardt GF. New renal scars in children with urinary tract infections, vesicoureteral reflux and voiding dysfunction: a prospective evaluation. J Urol. 1997;158(2): 566-568

3. van Gool JD, Hjälmås K, TamminenMöbius T, Olbing H; The International Reflux Study in Children. Historical clues to the complex of dysfunctional voiding, urinary tract infection and vesicoureteral reflux. J Urol. 1992;148(5 pt 2):1699-1702

4. Snodgrass W. Relationship of voiding dysfunction to urinary tract infection and vesicoureteral reflux in children. Urology. 1991;38(4): 341-344 
5. Seruca H. Vesicoureteral reflux and voiding dysfunction: a prospective study. J Urol. 1989;142 (2 pt 2):494-498, discussion 501

6. Keren R, Carpenter MA, Hoberman A, et al. Rationale and design issues of the Randomized Intervention for Children With Vesicoureteral Reflux (RIVUR) study. Pediatrics. 2008;122(suppl 5):S240-S250

7. Keren R, Shah SS, Srivastava R, et al; Pediatric Research in Inpatient Settings Network. Comparative effectiveness of intravenous vs oral antibiotics for postdischarge treatment of acute osteomyelitis in children. JAMA Pediatr. 2015;169(2):120-128

8. Farhat W, Bägli DJ, Capolicchio G, et al. The dysfunctional voiding scoring system: quantitative standardization of dysfunctional voiding symptoms in children. J Urol. 2000;164(3 pt 2):1011-1015

9. Shaikh N, Hoberman A, Wise B, et al. Dysfunctional elimination syndrome: is it related to urinary tract infection or vesicoureteral reflux diagnosed early in life? Pediatrics. 2003;112(5):1134-1137

10. Benninga M, Candy DC, Catto-Smith $A G$, et al. The Paris Consensus on Childhood Constipation Terminology (PACCT) Group. J Pediatr Gastroenterol Nutr. 2005;40(3):273-275

11. Schwab CW Jr, Wu HY, Selman H, Smith GH, Snyder HM III, Canning DA. Spontaneous resolution of vesicoureteral reflux: a 15-year perspective. J Urol. 2002;168 (6) :2594-2599

12. Soygür T, Arikan N, Yeşilli C, Göğüş 0 . Relationship among pediatric voiding dysfunction and vesicoureteral reflux and renal scars. Urology. 1999;54(5):905-908

13. Taylor CM, Corkery JJ, White RH. Micturition symptoms and unstable bladder activity in girls with primary vesicoureteric reflux. Br J Urol. 1982;54(5):494-498

14. Herndon CD, Decambre M, McKenna $\mathrm{PH}$. Interactive computer games for treatment of pelvic floor dysfunction. $J$ Urol. 2001;166(5):1893-1898 